\title{
Passive Covert Radars using CP-OFDM signals. A new efficient method to extract targets echoes
}

\author{
Gilles Chabriel *, Jean Barrère *, Ghislain Gassier*, Francoise Briolle ${ }^{\dagger}$ \\ *UTLN, CNRS-IM2NP, Bat. R, BP 132, F-83957 La Garde Cedex, France \\ Email: gilles.chabriel@im2np.fr, jean.barrere@im2np.fr, ghislain.gassier@im2np.fr \\ $\dagger$ Aix Marseille Université, Université de Toulon, CNRS, CPT, UMR7332, 13288 Marseille, France \\ Email: briolle@cpt.univ-mrs.fr
}

\begin{abstract}
Passive Coherent Location (PCL) systems use the signal transmitted by so called illuminators-of-opportunity in the environment to illuminates the targets. With CP-OFDM transmissions, it is quite easy to recover the original data and then to reconstruct the original transmission. The main difficulty is to extract, from the mixture received on the sensors, only the targets echoes. A new efficient and low complexity method is exposed here. Results on both simulated and real data are presented.
\end{abstract}

\section{INTRODUCTION}

A Passive Coherent Location (PCL) system or Passive Covert Radar is an emerging and highly promising technology, which may be used in many application areas in the future [1], [2], [3], [4].

A passive radar exploits one (bistatic case) or several (multistatic case) so called "illuminators-of-opportunity" in the environment to detect moving targets through their Doppler. The illuminators, normally used for radio or TV broadcasting, GSM transmissions, Wifi, etc. could be associated to a Passive Covert Radar[5], [6], [7].

The main advantages of such systems, with respect to conventional (active) radars, include low cost architecture, low energy requirement, detection of stealth targets and potentially null probability of interception.

Besides these incontestable practical advantages, a major problem is that Doppler echoes are in general more difficult to detect than with an active (monostatic) radar, since the bistatic angle is in general greater than a mono-static angle, and the target radar cross section (RCS) lower. Continual presence of the line of sight (LOS) emission with several orders of magnitude greater than the Doppler echoes may also bury the echoes of interest.

Reduction of the unwanted zero-Doppler echoes (defined here as "clutter") is then particularly important in such systems if one wants to expect an efficient target detection.

Nowadays broadcast transmissions are changing more and more from analog to digital. With CP-OFDM signals, widely used for communications transmissions as Digital Audio and Video Broadcasting Wifi transmissions, etc., it is possible to recover the original transmitted digital data and then, to synthesize a sampled version of the original transmitted signal. This signal will be used as a reference signal.

The main difficulty of PCL is to extract from the surveillance signal the very weak target's echoes which will be compared to the reference signal (direct path) in order to estimate the delay and the doppler effect.

After a presentation of Passive Covert Radar using CPOFDM and a brief presentation of the process to recover the original transmitted signal (SEC:II), a new efficient method to extract targets echoes from the clutter (SEC:III) and results are presented (SEC:IV) on simulated data and on real data.

\section{Position OF THE PROBLEM}

\section{A. Modelling}

A typical Passive Covert bi-static Radar scene is illustrated in Fig. 1, where a standard transmitter illuminates a scene composed of different static reflectors due to topography, buildings, ..., and one or several moving reflectors of interest or targets (here an aircraft). A receiving antenna collects the radiations from the $\mathrm{CP}-\mathrm{OFDM}$ transmitter and from the different reflectors.

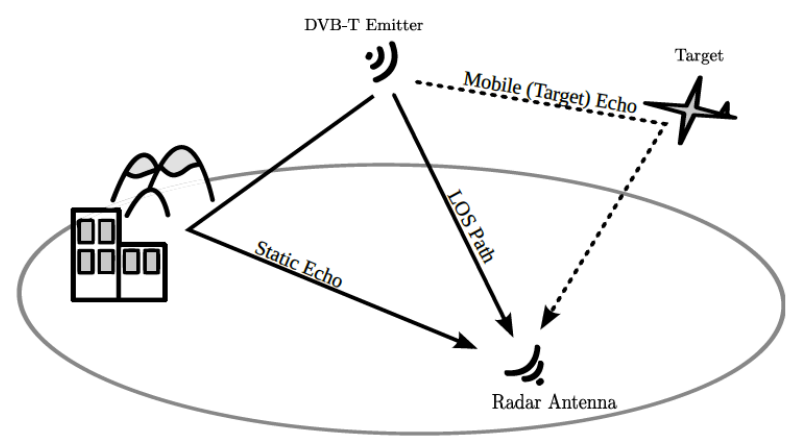

Fig. 1: Passive radar scene.

Usually, a passive radar is equipped with two receiving channels: reference and surveillance channels. The signal from the reference channel, is used as the original transmitted signal (direct path) and compared to the surveillance signal.

On the receiving antenna, we consider the baseband received signal $s(t)$ as a linear combination of the CP-OFDM emitted signal $s_{e}(t)$ following the direct path, and of its static and Doppler echoes corrupted by sensor noise. 
We can write:

$$
\begin{aligned}
s(t) & =\alpha s_{e}\left(t-t_{0}\right) \\
& +\sum_{i} \beta_{i} s_{e}\left(t-\tau_{i}-t_{0}\right) \\
& +\sum_{j} \gamma_{j} s_{e}\left(t-\tau_{j}-t_{0}\right) \exp \left(i 2 \pi f_{d_{j}}(t)\right)+\eta(t),
\end{aligned}
$$

where:

- $t_{0}$ is the propagation delay between the receiving antenna and the transmitter,

- $\tau_{i}+t_{0}$ and $\tau_{j}+t_{0}$ are the delays between the receiving antenna and respectively the $i^{\text {th }}$ static reflector and the $j^{\text {th }}$ target,

- $f_{d_{j}}$ is the frequency shift due to the radial velocity of the target $j$ (Doppler effect),

- $\alpha, \beta_{i}, \gamma_{j}$ are complex factors. $\beta_{i}$ and $\gamma_{j}$ are related to positions and RCS of the different reflectors.

- $\eta(t)$ is the sensor noise contribution assumed to be centered.

NOTA: the model of the baseband signal do not take into account the carrier frequency offset (CFO) effect due to the difference between the transmitter and receiver local oscillators. Different methods can be used to correct this effect ([10], [11], [12]).

Unlike conventional active radar systems, the original emitted signal $s_{e}(t)$ is not directly available. Nevertheless, with a CP-OFDM transmission, one can recover perfectly the transmitted data and consequently synthesize the baseband version of the emitted signal. This signal will be called the reference signal denoted by $s_{\text {ref }}(t)$. This process is detailed in II-B.

Then, the received signal can be written as

$$
\begin{aligned}
s(t) & =\alpha s_{\text {ref }}(t) \\
& +\sum_{i} \beta_{i} s_{\text {ref }}\left(t-\tau_{i}\right) \\
& +\sum_{j} \gamma_{j} s_{\text {ref }}\left(t-\tau_{j}\right) \exp \left(i 2 \pi f_{d_{j}} t\right)+\eta(t) .
\end{aligned}
$$

In practice, the target's radar cross section (RCS) is very weak and consequently $\left|\gamma_{j}\right|$ is very small compared to $|\alpha|$ and of most of $\left|\beta_{i}\right|$.

It is then necessary to enhance, from the received signal $s(t)$, the target's echoes signal

$$
s_{\text {tar }}(t)=\sum_{j} \gamma_{j} s_{\text {ref }}\left(t-\tau_{j}\right) \exp \left(i 2 \pi f_{d_{j}} t\right)
$$

or equivalently to reduce the unwanted echoes, denoted clutter

$$
s_{\text {clut }}(t)=\alpha s_{\text {ref }}(t)+\sum_{i} \beta_{i} s_{\text {ref }}\left(t-\tau_{i}\right)
$$

by a proper signal processing.

Finally, this ideally "clutter-cleaned" signal will be "compared" to the reference signal $s_{\text {ref }}(t)$ (ambiguity function) in order to estimate the radial speed and the bi-static distance of the different targets. Different methods have been proposed [8], [9]. The new efficient proposed method here will be based on the property of CP-OFDM communications that is a precise estimation of the propagation channel which allow echoes cancellation.

\section{B. Construction of the reference signal}

The structure of CP-OFDM signal allows a simplified correction of the multipath effect in order to recover the original transmitted data. Indeed, with such a signal, it is possible to get an accurate estimation of the propagation channel and then, by inverse filtering, to get the original data. These data will be used to synthesize a sampled version of the original signal, used as a reference signal. Let us briefly recall the relevant characteristics of a CP-OFDM emission.

1) CP-OFDM signals: CP-OFDM signal relies on an orthogonal frequency division multiplexing (OFDM), which consists of a modulation method of closely spaced orthogonal sub-carriers. The OFDM analog signal is a sequence of symbols. Each symbol, of duration $T_{s}$, is composed of $K$ orthogonal sub-carriers $\nu_{k}$ carrying the complex data $d_{k}^{m}$. A data can only take $M$ different numerical complex values depending on the $M$-array QAM modulation used.

A symbol consists of two parts: a useful part of duration $T_{u}$ (carrying the data) and a redundant part of duration $\Delta$ so-called the cyclic prefix $(\mathrm{CP})$. The cyclic prefix $(\mathrm{CP})$ is obtained by copying the last part of the symbol and attaching it in front of the useful part. $\mathrm{CP}$ allows to eliminate inter symbol (ISI) and inter carrier (ICI) interferences induced by significant echoes of delay less than $\Delta$. Note that orthogonality (on the duration $T_{u}$ ) between any pair of sub-carriers requires that $\nu_{k}=\frac{k}{T_{u}}$.

Assuming that the transmitter time origin is taken at the emission of the first symbol $(m=0)$, we can express the useful part of the $m^{\text {th }}$ emitted symbol as:

$s_{e}^{m}(t)=\sum_{k=0}^{K-1} d_{k}^{m} \exp \left(2 i \pi \frac{k}{T_{u}} t\right) \quad m T_{s}+\Delta<t \leq(m+1) T_{s}$.

2) Estimation of the propagation channel: to simplify the exposure, let us initially consider a noise-free case without echoes from mobile targets. The received signal is then the sum of the direct path and echoes from static reflectors. After synchronization onto the first emitted symbol and CP elimination, the received signal corresponding the $m^{\text {th }}$ emitted symbol can be written as a function of time, for the duration $T_{u}$, as

$s^{m}(t)=\sum_{k=0}^{K-1} \tilde{d}_{k}^{m} \exp \left(2 i \pi \frac{k}{T_{u}} t\right), \quad m T_{s}+\Delta<t \leq(m+1) T_{s}$,

where $\tilde{d}_{k}^{m}=H^{m}\left(\nu_{k}\right) d_{k}^{m}$ is the transmitted data (complex amplitude of the $k^{\text {th }}$ sub-carrier). The unknown coefficient 
$H^{m}\left(\nu_{k}\right)$ is the frequency response of the propagation channel at the frequency $\nu_{k}$ at the time $m T_{s}$. Note that under a block-fading channel assumption, one can consider that $H^{m}\left(\nu_{k}\right)$ remains constant over several symbols.

Let us sample the signal $s^{m}(t)$ at the frequency $\frac{K}{T_{u}}$, given by the CP-OFDM standard; we obtain the following numerical sequence:

$$
s^{m}[n]=\sum_{k=0}^{K-1} \tilde{d}_{k}^{m} \exp \left(2 i \pi \frac{k n}{K}\right), \quad 0 \leq n \leq K-1 .
$$

The coefficients $\tilde{d}_{k}^{m}$ are then obtained by discrete Fourier transform through a fast Fourier transform algorithm (FFT):

$$
\tilde{d}_{k}^{m}=\sum_{n=0}^{N-1} s^{m}[n] \exp \left(-2 i \pi \frac{k n}{K}\right), \quad 0 \leq k \leq K-1 .
$$

Most of CP-OFDM standard, like DVB, requires that, inside a symbol $m, P$ particular sub-carriers $\nu_{p}$ called pilot frequencies, carry the fixed data $\left\{d_{p}^{m}\right\}_{p}$ see e.g. [13].

Now, assuming that the coefficients $\left\{d_{p}^{m}\right\}_{p, m}$ are known, one can compute the frequency response $\left\{H^{m}\left(\nu_{p}\right)\right\}_{p, m}$ of the propagation channel at the frequencies $\left\{\nu_{p}\right\}_{p, m}$ :

$$
H^{m}\left(\nu_{p}\right)=\frac{\tilde{d}_{p}^{m}}{d_{p}^{m}}, p \in 1, \cdots, P .
$$

The frequency response of the channel for the others frequencies will be estimated by interpolation and the estimation of the original data can be processed by inverse filtering,

$$
d_{k}^{m}=\frac{\tilde{d}_{k}^{m}}{H^{m}\left(\nu_{k}\right)}, k \in 0, \cdots, K-1
$$

Actually, both the presence of noise $\eta(t)$ and Doppler echoes $s_{\text {tar }}(t)$ introduce errors during this estimation process. However, when the signal-to-noise ratio is sufficient and when the levels $\left|\gamma_{i}\right|$ of the Doppler echoes are weak enough, mapping the estimated data in accordance with the QAM constellation allows to correct most of these errors. We will consider here that after this process, the data are perfectly recovered.

We can already note that if the original transmitted data are perfectly recovered, then the propagation channel can be estimated with a very strong accuracy, for each symbol.

3) The reference signal: applying an inverse fast Fourier Transform (IFFT) onto the sequence $\left\{d_{k}^{m}\right\}_{k}$ to obtain the useful part of the symbol and attaching in front the $\mathrm{CP}$ insertion, one obtains the sampled version $\left\{s_{r e f}^{m}[n]\right\}_{n}$ of the original transmitted CP-OFDM symbol $m$. A sequence of symbols $\left\{s_{r e f}^{m}[n]\right\}_{n, m}$ will provides the whole reference signal $\left\{s_{\text {ref }}\right\}$ we seek:

$$
s_{\text {ref }}=\left\{s_{\text {ref }}^{0}, s_{\text {ref }}^{1}, \cdots, s_{\text {ref }}^{m}, \cdots\right\} .
$$

\section{Clutter Removal Method}

The method will rely on the hypothesis that the effect due to the static reflectors (including the direct path), will be the "same over several symbols (the duration $T_{s}$ of a symbol is about $1 \mathrm{~ms}$ ).

Since, with CP-OFDM signals, it is possible to recover the original data with a great accuracy, it is also possible to estimate, with a great accuracy, the frequency response $H^{m}\left(\nu_{k}\right)$ of the propagation channel for each symbol $m$. If it is possible to estimate the propagation channel $H_{c}\left(\nu_{k}\right)$ of the clutter ( echoes from static reflectors and direct path), then it will be easy to synthesize the clutter: $S_{c l u t}\left(\nu_{k}\right)=H_{c}\left(\nu_{k}\right) . S_{r e f}\left(\nu_{k}\right)$. Then, the clutter will be removed from the surveillance signal to obtain only the target's echoes.

\section{A. Method}

from the general noisy case (II-A) involving clutter and Doppler echoes, the sampled version of the baseband received signal $\mathrm{s}(\mathrm{t})$ is:

$$
\{s\}=\left\{s_{\text {clut }}\right\}+\left\{s_{\text {tar }}\right\}+\{\eta\} .
$$

Now, considering any symbol $m$ without its $\mathrm{CP}$, we have

$$
s^{m}[n]=s_{\text {clut }}^{m}[n]+s_{\text {tar }}^{m}[n]+\eta^{m}[n], \quad 0 \leq n \leq K-1 .
$$

Under the block-fading channel assumption, $s_{c l u t}^{m}[n]$ is expressed as

$$
\left.s_{\text {clut }}^{m}[n]=\sum_{k=0}^{K-1} H_{c}\left(\nu_{k}\right) d_{k}^{m} \exp \left(2 i \pi \nu_{k}\right)\right), \quad 0 \leq n \leq K-1,
$$

where $H_{c}\left(\nu_{k}\right)$ is the channel frequency response due to the clutter, assumed to be constant for the $\mathrm{M}$ symbols.

Computing the FFT of the $m^{\text {th }}$ received symbol $s^{m}[n]$, one obtains

$$
S^{m}[k]=\tilde{d_{k}^{m}}=H^{m}\left(\nu_{k}\right) d_{k}^{m}
$$

where $\tilde{d}_{k}^{m}$ are the received data and $d_{k}^{m}$ are the original one. $H^{m}\left(\nu_{k}\right)$ is the frequency response of the propagation channel for the symbol $m$, given by

$$
H^{m}\left(\nu_{k}\right)=\frac{\tilde{d_{k}^{m}}}{d_{k}^{m}}
$$

The FFT of the $m$-th received symbol can also be written, for $0 \leq k \leq K-1$, as

$$
S^{m}[k]=H^{m}\left(\nu_{k}\right) d_{k}^{m}=H_{c}\left(\frac{k}{T_{u}}\right) d_{k}^{m}+S_{\text {tar }}^{m}[k]+N^{m}[k],
$$

where $S_{\text {tar }}^{m}[k]$ and $\eta^{m}[k]$ are respectively the discrete Fourier transform of $s_{\text {tar }}^{m}[n]$ and $\eta^{m}[n]$.

We can divide the previous equation (Eq.2) by $d_{k}^{m}$ to get

$$
\hat{H}^{m}\left(\nu_{k}\right)=H_{c}\left(\nu_{k}\right)+\frac{S_{\text {tar }}^{m}[k]}{d_{k}^{m}}+\frac{N^{m}[k]}{d_{k}^{m}}, \quad 0 \leq k \leq K-1
$$


One can demonstrate that

$$
\hat{H}_{c}\left(\nu_{k}\right)=\frac{1}{M} \sum_{m=0}^{M-1} \hat{H}^{m}\left(\nu_{k}\right)
$$

is an unbiased estimator of the channel frequency response $H_{c}\left(\nu_{k}\right)$ due to the clutter effect.

From $\hat{H}_{c}\left(\nu_{k}\right)$ one can obtain an estimation of $S_{t a r}^{m}[k]$ by computing

$$
\hat{S}_{\text {tar }}^{m}[k]=S^{m}[k]-\hat{H}_{c}\left(\nu_{k}\right) \cdot S_{r e f}[k] .
$$

\section{B. Process}

The different stages of the proposed frequency clutter removal method can be found in Fig. 2.

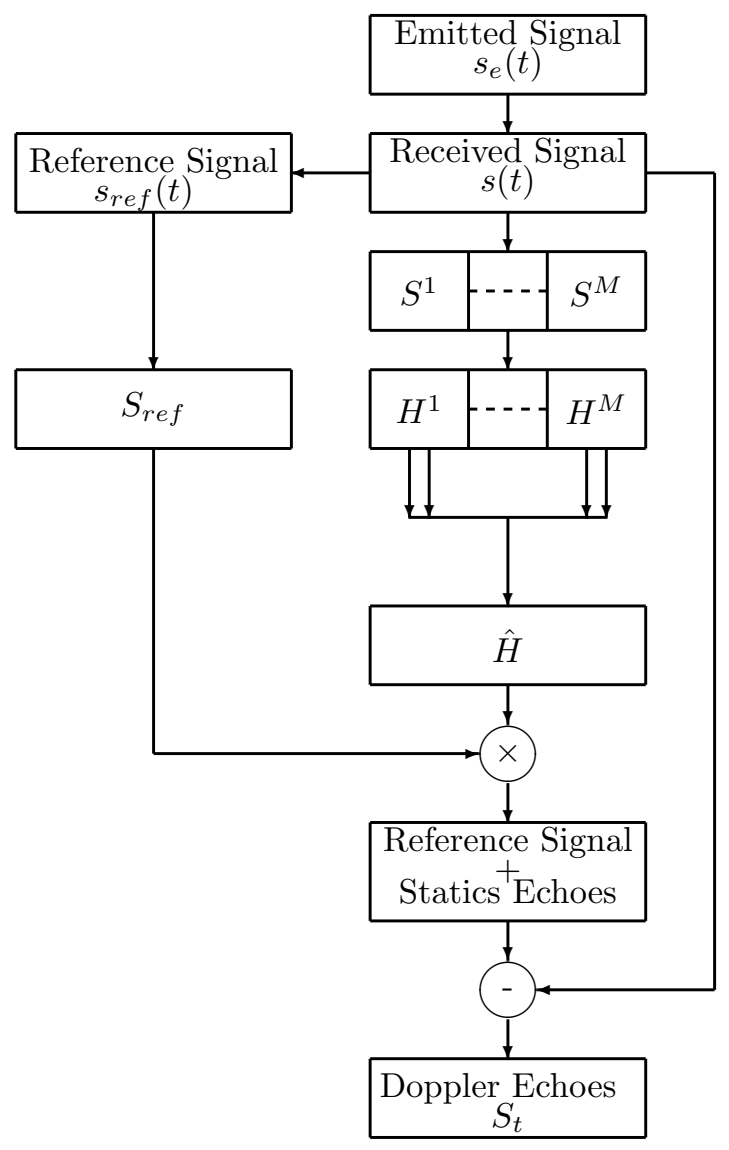

Fig. 2: Frequency clutter removal method.

\section{RESULTS}

\section{A. Simulated data}

We use 90 symbols of an original CP-OFDM signal, encoded with the DVB-T standard (8K, QAM-64, $T_{\Delta}=T_{u} / 16$ ). The sensors noise is a white gaussian noise and the signal to noise ratio is equal to $40 \mathrm{~dB}$.
The echoes from 100 static reflectors are added to the original CP-OFDM signal, delayed randomly by $\tau_{i}$ and amplified by $\beta_{i}$ such as $\tau_{i}<T_{\Delta}$ and $20 \log _{10}\left(\frac{\alpha}{\beta_{i}}\right)<-30 d B$, with $\alpha$ the amplitude of the original signal. The echoes direction (phase) is random.

The echoes of 3 targets are added to this mixture:

Target \#1 : distance to the receptor $10 \mathrm{~km}$, doppler effect 20 $\mathrm{Hz}$ and $\frac{\alpha}{A_{1}}=-50 \mathrm{~dB}$, with $A_{1}$ the echo's amplitude,

Target \#2 : distance to the receptor $12,5 \mathrm{~km}$, doppler effect $125 \mathrm{~Hz}$ and $\frac{\alpha}{A_{2}}==-50 \mathrm{~dB}$, with $A_{2}$ the echo's amplitude, Target \#3 : distance to the receptor $30 \mathrm{~km}$, doppler effect -150 $\mathrm{Hz}$ and $\frac{\alpha}{A_{3}}==-60 \mathrm{~dB}$, with $A_{3}$ the echo's amplitude.

Two of the targets are very close to the PCL receiver (12 $\mathrm{km}$ and $12.5 \mathrm{~km})$. One of them moves very slowly, with a doppler echo equal to $20 \mathrm{~Hz}$. The third target is far from the receiver $(30 \mathrm{~km})$ and the attenuation of the echo is $60 \mathrm{~dB}$.

The parameters of the simulation are presented in the table below.

\begin{tabular}{|c|c|c|c|}
\hline & Dop. freq. $(\mathrm{Hz})$ & At. $(\mathrm{dB})$ & Range $(\mathrm{km})$ \\
\hline Echoes (100) & 0 & $>30$ & $<16$ \\
\hline Target \#1 & 20 & 50 & 10 \\
\hline Target \#2 & 125 & 50 & 12,5 \\
\hline Target \#3 & -150 & 60 & 30 \\
\hline
\end{tabular}

1) Results: the representation of the ambiguity function, [14], [15], defined as

$$
\chi(x, \nu)=\int_{-\infty}^{+\infty} s_{\text {ref }}(t) s_{\text {tar }}^{*}(t-x / c) e^{2 i \pi \nu t} d t
$$

will give an image ( doppler frequency versus bi-static distance) where the maxima of the ambiguity function the target will be plotted in red.

Two methods were used : our new method (presented in SEC:III) and the least squares based method proposed by Colone et al. [8], called ECA-batches, where the signal was cut in thirty parts of three symbols each. The results are presented in fig. 3 and 4.

The results obtained with the new efficient method allow to detect the three targets on the simulated data, even when the target is moving slowly (target \#2). Withthe ECA-batches method it was impossible to detect this target, which was seen as a clutter (zero doppler) echo.

\section{B. Real data}

The campaign of measurements was achieved using illuminators in a Single Frequency Network. The broadcast signal, encoded with DVB-T standard (8K, QAM-16, $T_{\Delta}=T_{u} / 16$, is modulated at the frequency of $514 \mathrm{MHz}$. The receiver was an array composed of 16 sensors located close to an airport and an highway.

The cross-ambiguity function (extended to the multi-sensor case) presented in figure 5 is obtained from 82 OFDM symbols. 


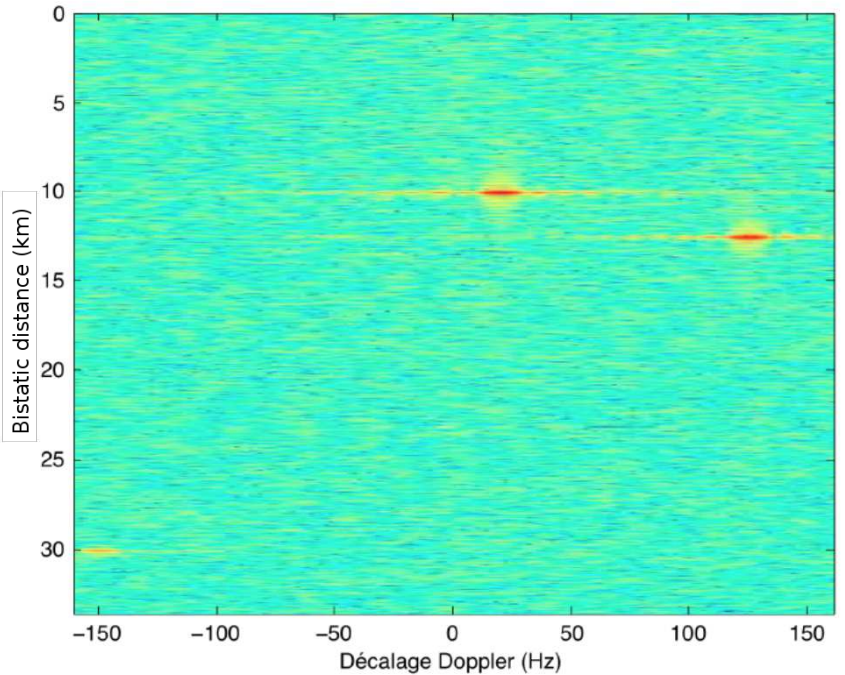

Fig. 3: New efficient method - ambiguity function

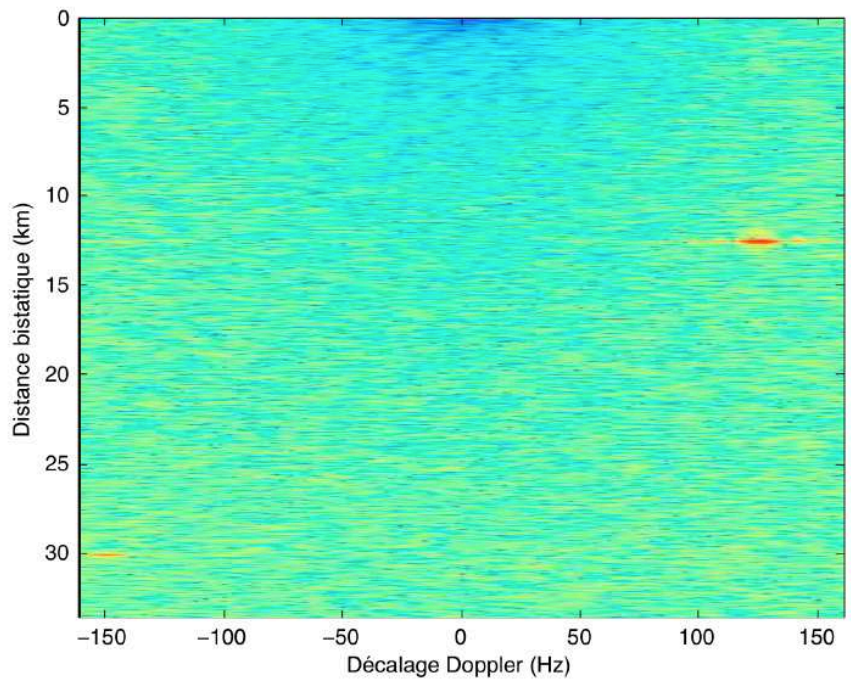

Fig. 4: ECA-batches method - ambiguity function

Two kind of detection can be distinguished : firstly, one can see a set of targets detected in a single area characterized by a small Doppler, located at about $12 \mathrm{~km}$; secondly, isolated detection peaks characterized by higher Doppler are observed. The first ones are interpreted as a consequence of the highway traffic, the second ones are associated to the air traffic.

A result of "tracking" is presented fig. 6 where 155 ambiguity functions were computed and merge together in order to have an image of the context during 155 seconds.

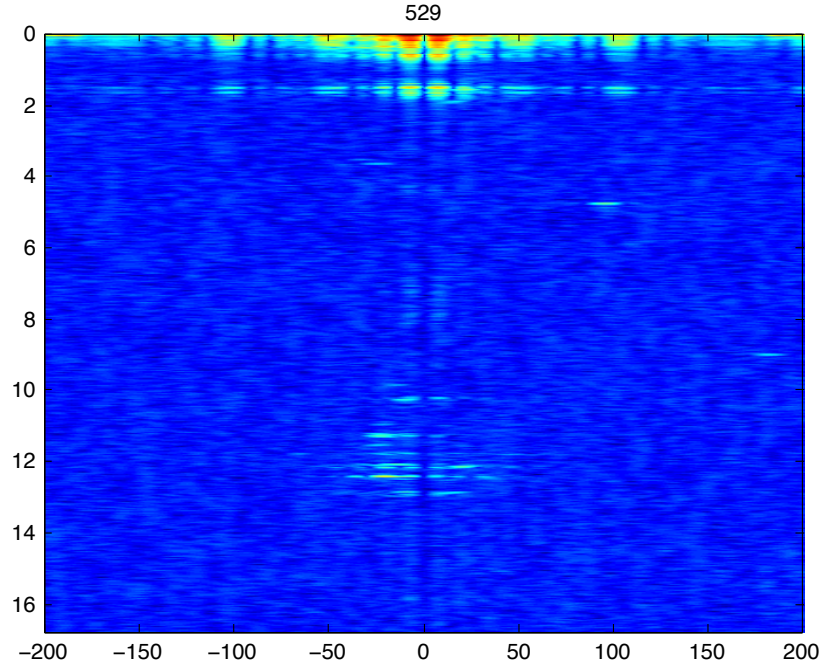

Fig. 5: New efficient method - ambiguity function

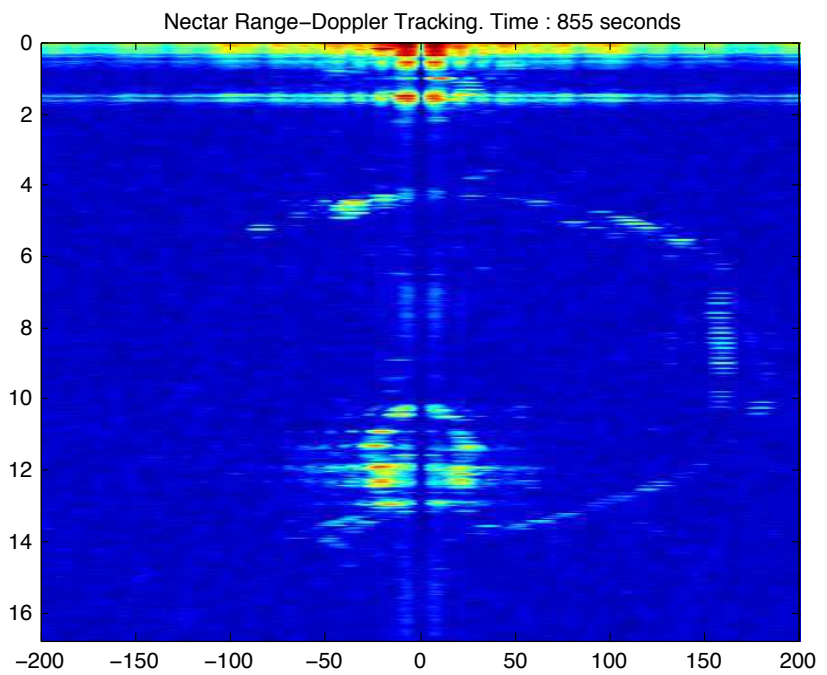

Fig. 6: Tracking with a new efficient method

\section{CONClusion}

The method presented here seems to be promising. First, because its implementation is very simple and requires few computation. The results on simulated data show that the method is reliableand results on real data are hopeful.

\section{ACKNOWLEDGMENT}

The campaign of measurements was achieved by Thales Air Systems, ONERA, DGA (Direction générale de l'armement) and CEAM (Centre d'Expériences Aériennes Militaires) jointly with the BWB (Bundesamt fur Wehrtechnik und Beschaffung) and Fraunhofer Institute. We are particularly grateful to J. Colin, from the DGA, for giving us access to the data. 


\section{REFERENCES}

[1] H. Kuschel and D. O'Hagan,Passive radar from history to future, in Radar Symposium (IRS), 2010 11th International (pp. 1-4). IEEE, 2010.

[2] J.M. Thomas, C.J. Baker and H.D. Griffiths, $H F$ passive bistatic radar potential and applications for remote sensing, in New Trends for Environmental Monitoring Using Passive Systems, 2008 (pp. 1-5). IEEE, 2008.

[3] J. Song, Y. He, F.Q Cai, and X.M. Tang, Overview of passive radar technology based on non-cooperative radar illuminator. Systems Engineering and Electronics, 31(9), 2151-2156, 2009.

[4] H.D. Griffiths, and C.J. Baker, C. J. Passive coherent location radar systems. Part 1: Performance prediction in Radar, Sonar and Navigation, IEE Proceedings- Vol. 152, No. 3, pp. 153-159. IET, 2005.

[5] P. Falcone, F. Colone, C. Bongioanni, C.and P. Lombardo, Experimental results for OFDM WiFi-based passive bistatic radar in Radar Conference, 2010 IEEE (pp. 516-521). IEEE, 2010.

[6] H. Sun, D.K. Tan,Y. Lu, and M. Lesturgie, Applications of passive surveillance radar system using cell phone base station illuminators. Aerospace and Electronic Systems Magazine, IEEE, 25(3), 10-18, 2010

[7] X. Neyt,J. Raout, M. Kubica, V. Kubica, S. Roques, M. Acheroy, M. and J.G. Verly, Feasibility of STAP for passive GSM-based radar in Radar, 2006 IEEE Conference on (pp. 6-pp). IEEE, 2006.

[8] F. Colone, D.W. O'hagan, P. Lombardo, and C.J. Baker, C. A multistage processing algorithm for disturbance removal and target detection in passive bistatic radar Aerospace and Electronic Systems, IEEE Transactions on, 45(2), 698-722, 2009.

[9] D. Poullin, Passive Radar using COFDM (DAB or DVB-T) Broadcasters using as Opportunistic Illuminators, in Special Issue of IEE Proc. Radar, Sonar and Navigation on Passive Radar Systems, Vol. 152, No. 3, pp 143-152, 2008

[10] M. Sliskovic, Carrier and sampling frequency offset estimation and correction in multicarrier systems, in Global Telecommunications Conference, 2001. GLOBECOM'01. IEEE, Vol. 1, pp. 285-289, 2001

[11] E.S Shim, S.T Kim, H. K. Song, and Y. H. You, OFDM carrier frequency offset estimation methods with improved performance, Broadcasting, IEEE Transactions on, 53(2), pp. 567-573, 2007

[12] Y.H. You, S.T. Kim, K.T. Lee, and H.K. Song, An improved sampling frequency offset estimator for OFDM-based digital radio mondiale systems, Broadcasting, IEEE Transactions on, 54(2), pp. 283-286, 2008.

[13] ETSI Standard : EN 300744 V1.5.1 Digital Video Broadcasting (DVB).

[14] Woodward, P. M., Probability and information theory, with applications to radar, London : Pergamon Press, 1953.

[15] Le Chevalier, F. Principles of radar and sonar signal processing, Artech House, 2002. 